\title{
The Relationship Between the BRAFV600E Mutation in Differentiated Thyroid Cancer and Clinicopathologic Factors in Vietnam
}

\author{
Mai Trong Khoa, Pham Cam Phuong*, Nguyen Thanh Trung, Nguyen Tien Lung, Nguyen Thuan Loi and Tran Dinh \\ Ha
}

The Gene Stem Cell Unit the Nuclear Medicine and Oncology center, Bach Mai hospital, Vietnam

Received: 制: September 11, 2018; Published: 制 September 17, 2018

*Corresponding author: Pham Cam Phuong, The Gene - Stem Cell Unit, The Nuclear Medicine and Oncology Center, Bach Mai Hospital, Hanoi, Vietnam

\section{Abstract}

Objective: Among more than 40 mutations identified in the BRAF gene, the BRAF T1799A (V600E) point mutation is the most common and accounts for more than $90 \%$ of all the mutations found in the BRAF gene. It has been found to occur frequently in thyroid cancer. While some reports suggest the BRAF V600E mutation is associated with poor prognosis and recurrence or iodine uptake resistance, this remains a controversial issue. The aim of study was to: 1) determine the status of BRAF mutation in differentiated thyroid cancer in Bach Mai hospital; 2) evaluate the association of the BRAF mutation with clinicopathological parameters.

Patients and Methods: Retrospective analysis of the BRAF V600E mutation in differentiated thyroid cancer in 198 patients from November 2015 to March 2017 at Bach Mai hospital using BRAF 600/601 Strip Assay® (ViennaLab, Austria). The results were correlated with clinicopathological factors.

Results: The BRAF V600E mutation was detected in 150 cases (75.8\%). BRAF mutation was found in 69/92 (75\%) patients having metastasis. Mutation was found in distant metastasis, lymph node metastasis and in both distant-lymph node metastasis: $63 / 84(75 \%), 9 / 13$ (69.2\%) and $3 / 5(60 \%)$, respectively. Among differentiated thyroid cancer, BRAF mutation was detected in follicular-papillary thyroid cancer (80\%), papillary thyroid cancer (76.6\%) and in follicular thyroid cancer (40\%). There was no significant correlation between the presence of BRAF mutation and clinicopathological factors. Conclusions: The BRAF mutation rate in thyroid cancer patients is high, especially for follicular-derived papillary and papillary thyroid cancer. The lack of a statistically significant association of BRAF and clinical characteristics indicates the need of conducting research with larger sample sizes in the future.

\section{Introduction}

Thyroid cancer shows the fastest rising incidence among all major human cancers, currently with 44,670 new cases in the United State. Differentiated thyroid cancer is the most common cancer of the endocrine system and the papillary thyroid cancer is the most common type, followed by follicular thyroid carcinoma. In recent decades, the incidence of this type of cancer has increased in Vietnam. According to GLOBOCAN 2012, the prevalence rate is $2.5 / 100000$ people, mortality rate is $1.3 / 100000$ people per year. Most thyroid cancers generally have a good prognosis after appropriate treatment including surgical procedure and radioiodine therapy [1]. However, the recurrence rate of differentiated thyroid cancer increased up to $30 \%$, and the cancer death rate was $8 \%$ after initial treatment at 30 years of follow-up. Studies in the world have shown that the primary cause of recurrence and metastasis in patients treated radioiodine therapy are low absorption and poor iodine intake (Table 1) [1,4].

Table 1: Sociodemographic characteristics of patients $(n=198)$.

\begin{tabular}{|c|c|c|c|}
\hline \multicolumn{2}{|c|}{ Sociodemographic characteristics of patients } & n & Proportion (\%) \\
\hline \multirow{3}{*}{ Age } & $<45$ & 96 & 48.5 \\
& $\geq 45$ & 102 & 51.5 \\
& Median & 45.1 & $16-76$ \\
\hline Age group & Range & 25 & 12.6 \\
\hline
\end{tabular}




\begin{tabular}{|c|c|c|c|}
\hline & 30 years old and over & 173 & 87.4 \\
\hline \multirow{2}{*}{ Gender } & Male & 26 & 13.1 \\
& Female & 172 & 86.9 \\
Sampling method & Surgery & 189 & 95.4 \\
& Biopsy & 9 & 4.6 \\
Sampling locations & Thyroid & 169 & 13.1 \\
& Nodal metastasis & 26 & 1.5 \\
\hline
\end{tabular}

Comment: Of the 198 patients participated in the study, there were 172 women and 26 men, with an average age of 45.1 years old, in which patients aged 45 years or older accounted for a higher proportion (51.5\%) Most patients (189/198 - 95.4\%) underwent primary tumor resection and histopathological examination with tissue samples taken from surgery. The primary sampling site was thyroid gland which accounted for $85.4 \%, 1.5 \%$ of sampling site was (3 patients) from distant metastatic tissue.

Activation of MAPK pathway by BRAF V600E was believed to play a dominant role in the development and progression of thyroid cancer[2]. Effectors of MAPK signaling pathway representing an early molecular event in papillary thyroid cancer regulate several genes related to cell proliferation, differential and survival. Gene expression analyses using DNA microarrays showed that different transcriptional profiles were associated with BRAF, RET/PTC and RAS mutation groups in human papillary thyroid cancer [10]. The differential expression profiles between these genetic alternations may explain why BRAF mutation has more close correlations with poor clinicopathological features compared with RET/PTC and RAS mutation[3]. The mutant BRAF can stimulate constitutive signaling which bypasses the need for extracellular growth factors. Subsequently phosphorylation of downstream MER1/2 and ERK1/2 leads to the expression of a few specific genes involved in cell proliferation, differential, survival, tumorigenesis, and even the microenvironment promoting the process of epithelialmesenchymal transition [3-4].

BRAF V600E mutation in thyroid cancer has been proved to be associated with high-risk clinicopathological characteristics, tumor recurrence and reduced sensitivity of radioiodine therapy. Conventional factors that demonstrate the high-risk clinicopathological characteristics included increased age, male gender, larger tumor size, extrathyroidal invasion, local lymph node metastasis, distant metastasis, and advanced disease stages. Recent studies have showed that significant correlations of BRAF mutation with the reliable prognostic predictors such as extrathyroidal invasion, lymph nodal metastasis, and advanced TNM stage[6]. In this study, we focus on the association of BRAF mutation with clinicopathological characteristics and BRAF mutation as a therapeutic target of thyroid cancer [5-6].

\section{Patients and Methods}

\section{Patients}

Retrospective study on 198 patients who had been diagnosed with thyroid cancer at The Nuclear Medicine and Oncology Center of Bach Mai Hospital, from November 2015 to March 2017. Informed consent for the evaluation of BRAF V600E mutation was obtained from all patients (Table 2).

Table 2: Clinical characteristics of patients $(n=198)$.

\begin{tabular}{|c|c|c|c|}
\hline \multicolumn{2}{|c|}{ Characteristics } & \multirow{2}{*}{$\begin{array}{c}\mathbf{n} \\
107\end{array}$} & \multirow{2}{*}{$\begin{array}{c}\text { Proportion (\%) } \\
59.4\end{array}$} \\
\hline & I-II & & \\
\hline Disease staging & III-IV & 60 & 33.3 \\
\hline & $\mathrm{N} / \mathrm{A}$ & 13 & 7.2 \\
\hline \multirow{4}{*}{$\mathrm{T}$ staging } & $\mathrm{T} 1$ & 35 & 17.7 \\
\hline & $\mathrm{T} 2$ & 95 & 48 \\
\hline & $\mathrm{T} 3 / \mathrm{T} 4$ & 28 & 14.1 \\
\hline & $\mathrm{N} / \mathrm{A}$ & 40 & 20.2 \\
\hline \multirow{4}{*}{ Nodal metastasis } & N0 & 70 & 35.4 \\
\hline & N1 & 84 & 40.9 \\
\hline & $\mathrm{N} 2 / \mathrm{N} 3$ & 3 & 1.5 \\
\hline & $\mathrm{N} / \mathrm{A}$ & 44 & 22.2 \\
\hline \multirow{3}{*}{ Distant metastasis } & M0 & 143 & 74.7 \\
\hline & M1 & 13 & 6.6 \\
\hline & $\mathrm{N} / \mathrm{A}$ & 37 & 18.7 \\
\hline
\end{tabular}




\begin{tabular}{|c|c|c|c|}
\hline \multirow{3}{*}{ Histopathology } & papillary thyroid cancer & 188 & 95 \\
\hline & follicular thyroid cancer & 5 & 2.5 \\
\hline & papillary- follicular thyroid cancer & 5 & 2.5 \\
\hline \multicolumn{2}{|c|}{ Total } & 198 & 100 \\
\hline
\end{tabular}

Comment: Among 198 paticipated patients, 107 of them was in early stage (I-II), accounted for 59.4\%. Most patients were in the T2 stage $(48 \%)$, the remaining patients in the T1, T3/T4 and unknown stage with the rate of $17.7 \% ; 14.1 \%$ and $20.2 \%$, respectively. The proportion of patients with 1 and $2 / 3$ nodal metastasis were $40.9 \%$ and $1.5 \%$, respectively. 143 out of 198 patients did not develop distant metastases, accounting for $74.7 \%$, only $13 / 198$ patients were detected with distant metastatic, accounting for $6.6 \%$. In terms of tumor tissue histology, the majority of 188/198 tumors were papillary, and the rest was follicular (5 patients) and follicular-derived papillary (5 patients).

\section{DNA Extraction and the BRAF V600E Mutation Analysis}

We performed the BRAF V600E mutation analysis on paraffin embedded sections of primary tumors obtained after thyroidectomy. Genomic DNA was extracted from $10 \mu \mathrm{m}$ thick sections of formalin-fixed paraffin embedded tumor tissue blocks using the QiAamp DNA FFPE tissue kit (Qiagen, USA). The concentration and purity of the extracted DNA were determined by a Qubit 2.0 spectrophotometer (Thermo Fisher Scientific, USA). The extracted DNA was stocked at $-20^{\circ} \mathrm{C}$ until further use. The assays for the detection of the BRAFV600E mutation were performed using the BRAF 600/601 StripAssay ${ }^{\circledR}$ (ViennaLab, Austria). All reactions were done in 20- $\mu$ Lvolumes using template DNA, and PCR master mix. All needed reagents were included with the kit. The PCR reaction of was performed using a Mastercycler (Eppendrod, USA). PCR cycling conditions were a $10 \mathrm{~min}$ hold at $37^{\circ} \mathrm{C}$ followed by 35 cycles of $94^{\circ} \mathrm{C}$ for $60 \mathrm{sec}, 70^{\circ} \mathrm{C}$ for $50 \mathrm{sec}, 56^{\circ} \mathrm{C}$ for $50 \mathrm{sec}, 60^{\circ} \mathrm{C}$ for $60 \mathrm{sec}$, and $60^{\circ} \mathrm{C}$ for $3 \mathrm{~min}$ (Tables $3 \& 4$ ). The PCR products were hybridized with streptavidin-alkaline phosphatase specific probe and results analysis. Data is processed by SPSS 15.0 software [7].

Table 3: Rate of BRAF mutation.

\begin{tabular}{|c|c|c|}
\hline BRAF status & n & \multicolumn{2}{|c|}{ Proportion (\%) } \\
\hline Mutated & 150 & 25.8 \\
\hline Wild type & 48 & 24.2 \\
\hline Total & 198 & 100 \\
\hline
\end{tabular}

Comment: Of the total 198 patients, the BRAF V600E mutation was found in 150 patients, accounting for $75.8 \%$.

Table 4: BRAF V600E mutation and clinicopathologic factors of differentiated thyroid cancer patients.

\begin{tabular}{|c|c|c|c|c|c|c|c|}
\hline \multirow{2}{*}{\multicolumn{2}{|c|}{ Patients clinicopathology }} & \multicolumn{6}{|c|}{ BRAF V600E mutation } \\
\hline & & \multirow{2}{*}{\begin{tabular}{|l|} 
Yes \\
83 \\
\end{tabular}} & \multirow{2}{*}{$\begin{array}{l}\text { No } \\
19\end{array}$} & \multirow{2}{*}{$\begin{array}{c}\text { OR } \\
0.52 \\
\end{array}$} & \multirow{2}{*}{$\begin{array}{c}\mathbf{9 5 \%} \mathbf{C I} \\
0.27-1.02\end{array}$} & \multirow{2}{*}{\begin{tabular}{|l|} 
(\%) \\
81.5
\end{tabular}} & \multirow{2}{*}{$\frac{\mathbf{p}}{0.057}$} \\
\hline$A_{0}$ & $<45$ & & & & & & \\
\hline Age & $\geq 45$ & 67 & 29 & & & 69.8 & \\
\hline \multirow{2}{*}{ Gender } & Male & 19 & 7 & 1.18 & $0.46-2.98$ & 73.1 & 0.732 \\
\hline & Female & 131 & 41 & & & 76.2 & \\
\hline \multirow{2}{*}{ Sampling method } & Surgery & 144 & 45 & 0.63 & $0.15-2.60$ & 76.2 & 0.529 \\
\hline & Biopsy & 6 & 3 & & & 66.7 & \\
\hline \multirow{3}{*}{ Sampling locations } & Thyroid & 127 & 42 & - & - & 75.2 & 0.562 \\
\hline & Nodal metastasis & 21 & 5 & & & 80.8 & \\
\hline & Distant metastasis & 2 & 1 & & & 66.8 & \\
\hline \multirow{3}{*}{ Histopathology } & Papillary & 144 & 44 & - & - & 76.6 & 0.243 \\
\hline & Follicular & 2 & 3 & & & 40.0 & \\
\hline & Papillary- follicular & 4 & 1 & & & 80.0 & \\
\hline \multirow{2}{*}{ Stage } & I-II & 80 & 27 & 1.22 & $0.57-2.59$ & 74.8 & 0.605 \\
\hline & III-IV & 47 & 13 & & & 78.3 & \\
\hline \multirow{3}{*}{ T staging } & $\mathrm{T} 1$ & 29 & 6 & - & - & 82.9 & 0.52 \\
\hline & $\mathrm{T} 2$ & 71 & 24 & & & 74.7 & \\
\hline & $\mathrm{T} 3 / \mathrm{T} 4$ & 20 & 8 & & & 71.4 & \\
\hline \multirow{2}{*}{$\mathrm{N}$ staging } & No & 53 & 17 & - & - & 75.7 & 0.8 \\
\hline & N1 & 60 & 21 & & & 74.1 & \\
\hline
\end{tabular}

Cite this article: Mai Trong K, Pham Cam P, Nguyen T T, Nguyen Tien L, Nguyen Thuan L, Tran Dinh H. The Relationship Between the BRAFV600E Mutation in Differentiated Thyroid Cancer and Clinicopathologic Factors in Vietnam. Biomed J Sci\&Tech Res 9(1)- 2018. BJSTR. 


\begin{tabular}{|c|c|c|c|c|c|c|c|}
\hline & $\mathrm{N} 2 / \mathrm{N} 3$ & 3 & 0 & & & 100 & \\
\hline \multirow{2}{*}{ M staging } & M0 & 113 & 35 & 0.68 & $0.2-2.4$ & 76.4 & 0.813 \\
\hline & M1 & 9 & 4 & & & 69.2 & \\
\hline
\end{tabular}

Comment: The results of the study show no correlation between BRAF mutagenicity characteristics such as age, sex, sampling method, sampling location, disease staging, T-stage, Nodal metastasis, distant metastasis and histopathological characteristics ( $\mathrm{p}>0.05)$.

\section{Result}

\section{Patients' Characteristics}

Tables $1 \& 2$

\section{The Status of BRAF Mutation}

Table 3

\section{The Relationship between the BRAFV600E Mutation in differentiated Thyroid Cancer and Clinicopathologic Factors}

Table 4

\section{Discussion}

In our study, the majority of patients were female, accounting for $86.9 \%(172 / 198)$, while the rate of male patients was $13.1 \%$ (26/198). Female/male ratio was 6,6/1 (172/26). The average age for both men and women were 45.1 years. These findings are in accordance with some findings to other studies: Nguyen Quang Truong (2013) reported that the proportion of male and female patients were $17.5 \%$ and $82.5 \%$, respectively; the rate of female/male was $4.7 / 1(113 / 24)[7]$. This study also found that the average age of both men and women is 39.7 years old. In the study conducted by [1-8], $76.3 \%$ of patients was female which was 3 times higher than male patients (23.7\%), the average age was 40.9 \pm 14.1 . The most common age group was from 31 to 45 years old, accounted for $40.1 \%$ of total patients while the high-risk age group (under 15 and over 45) accounted for 31.8\% [8]. In general, the incidence of thyroid cancer in women is three to five times higher than in men and is more likely to occur in young people. In terms of histopathological classification, our study showed that $95 \%$ of thyroid cancer patients was papillary form[9]. Similar studies conduted by Le Van Quang (2002)[4] among 308 cancer patients in the Vietnam nation cancer hospital discoverd that $79.5 \%$ thyroid cancer was papillary form, only $15.6 \%$ was in folicular form. Our study also like what is reported by Lim L.H và collegues (2002). Among 198 thyroid cancer patients, 78\% of them was papillary form, $19 \%$ of them was papilary form, $1 \%$ medullary thyroid carcinoma and $2 \%$ undifferentiated thyroid cancer [10].

This similarity suggests that papillary form remains a common histopathology of thyroid cancer. The results of many international researches reported the wide range of BRAF mutations frequency which was from $39 \%$ to $84 \%$. The frequency of BRAF mutation in our study was $75.8 \%$ and all mutations were detected as V600E which like the results of Kim SJ et al. (2012) in Korean patients (71\%). In addition, our results showed the high rate of BRAF mutation in the group of under 45 years (81.4\%), which is $12 \%$ higher in comparision with the group of over 45 years old. BRAF mutations were more common in women than in men with a mutation rate of $73.1 \%$ and $76.2 \%$, respectively. In our study, the BRAF mutations occurred more frequently in patients with papillary and follicularderived papillary form with mutation rates of $76.60 \%$ and $80 \%$, respectively. Patients with disease progression stage (III / IV) had a $4 \%$ higher of BRAF mutation rate in comparison with early stage patients (I/II). Regarding the $\mathrm{T}$ staging, nodal metastasis and distant metastasis, there was $8-11 \%$ difference between T1 stage and other T staging; $100 \%$ of patients with 2 or 3 nodal metastases had BRAF mutation. The rate of BRAF mutations in non-metastatic patients was $76.4 \%$ and was $5 \%$ higher than in patients with distant metastases. In term of sampling method, our results demonstrated that he detection rate of mutations in surgical specimens was $76.19 \%, 10 \%$ higher than that of biopsy specimens.

For the sampling site, the specimen collected at the nodal metastasis had the highest BRAF mutation rate $(80.8 \%)$, followed by thyroid site and distant metastatic with mutation rate of $75.2 \%$ and $66.7 \%$, respectively. Although our study found a difference in BRAF mutations in every clinical characteristic, but the results of the $\chi 2$ test showed no significant difference ( $p>0.05)$. These results suggest that there is no significantly association between BRAF mutations and some specific clinical characteristics such as age, sex, stage of disease, histopathological result, sampling sites and sampling methods, which are in accordance with other similar sample sizes research in the world. However, other studies performed on larger sample sizes, resulting in contradictory findings. This difference may be due to insufficient sample sizes and limited follow-up. Therefore, the implementation of future studies with larger sample sizes is critical to assess the prognosis of BRAF mutations in thyroid cancer patients. In addition, our study reported that the sampling site and sampling method did not have any statistically significant association with BRAF mutation ( $p>$ 0.05). This result has once again confirmed that the BRAF V600E mutation are unaffected by the sampling method and site.

\section{Conclusion}

Of the 198 thyroid patients in our study:

a) BRAF mutations were detected in 150/198 patients (75.76\%), with all mutations at codon 600 exon 15 (V600E).

b) BRAF mutation does not depend on age, gender, sampling methods, sampling sites, histopathological features, the stage of disease, T staging, Nodal metastasis and Distant metastasis.

c) The implementation of future studies with larger sample sizes is critical to assess the prognostic potential of BRAF mutations in thyroid cancer patients. 


\section{References}

1. ME Cabanillas, A Patel, BP Danysh, R Dadu, S Kopetz, et al. (2015) BRAF Inhibitors: Experience in Thyroid Cancer and General Review of Toxicity. Hormones and Cancer 6(1): 21-36.

2. Nikiforova MN, RS Wald AI, Roy S, Durso MB, Nikiforov YE (2013) Targeted next-generation sequencing panel (ThyroSeq) for detection of mutations in thyroid cancer. J Clin Endocrinol Metab 98(11): 1852-1860.

3. Elisei R, Viola D, TL, Giannini R, Romei C, et al. (2012) The BRAF(V600E) mutation is an independent, poor prognostic factor for the outcome of patients with low-risk intrathyroid papillary thyroid carcinoma: singleinstitution results from a large cohort study. J Clin Endocrinol Metab 97(12): 4390-4398.

4. Li C, Aragon Han P, L KC, Lee LC, Fox AC, et al. (2013) Does BRAF V600E mutation predict aggressive features in papillary thyroid cancer?

ISSN: 2574-1241

DOI: $10.26717 / B J S T R .2018 .09 .001748$

Pham Cam Phuong. Biomed J Sci \& Tech Res

(C) This work is licensed under Creative

Submission Link: https://biomedres.us/submit-manuscript.php
Results from four endocrine surgery centers. J Clin Endocrinol Metab 98(9): 3702-3712.

5. Mark Yarchoan, Virginia A Li Volsi, MS Brose (2015) BRAF Mutation and Thyroid Cancer Recurrence. Journal of Clinical Oncology 33(1): 7-8.

6. Xing M, Alzahrani AS, C KA, Viola D, Elisei R, et al. (2013) Association between BRAF V600E mutation and mortality in patients with papillary thyroid cancer. JAMA 309(14): 1493-1501.

7. Nguyen Quang Truong (2013) Study on the treatment of the thyroid cancer by surgical removal of the thyroid gland and thyrotoxic dose. Journal of the Military medicine pp. 102-106.

8. Tran Trong Kiem (2008) Study on the treatment of the thyroid cancer by surgical removal of the thyroid and I-131. Journal of the Military medicine p. 15-21.

$\begin{array}{ll}\text { BIOMEDICAL } & \text { Assets of Publishing with us } \\ \text { RESEARCHES } & \text { - Global archiving of articles } \\ \text { ISSN: 2574-1241 } & \text { - Immediate, unrestricted online access } \\ & \text { - Rigorous Peer Review Process } \\ \end{array}$

